\title{
Development of a Transformation Method for Metschnikowia borealis and other CUG-Serine Yeasts
}

\author{
Zachary B. Gordon ${ }^{1,2}$, Maximillian P.M. Soltysiak ${ }^{3}$, Christopher Leichthammer ${ }^{1}$, \\ Jasmine A. Therrien ${ }^{1}$, Rebecca S. Meaney ${ }^{1}$, Carolyn Lauzon ${ }^{1}$, Matthew Adams ${ }^{1}$, \\ Dong Kyung Lee ${ }^{3}$, Preetam Janakirama ${ }^{1}$, Marc-André Lachance ${ }^{3}$ and Bogumil J. Karas ${ }^{1,2, *(D)}$ \\ 1 Designer Microbes Inc., London, ON N6G 4X8, Canada; zgordon2@uwo.ca (Z.B.G.); cleichth@uwo.ca (C.L.); \\ jasmine.alyssa.therrien@gmail.com (J.A.T.); rmeaney2@uwo.ca (R.S.M.); carolyn.lauzon@gmail.com (C.L.); \\ adams.mil@hotmail.com (M.A.); preetam.janakirama@gmail.com (P.J.) \\ 2 Department of Biochemistry, Schulich School of Medicine and Dentistry, University of Western Ontario, \\ London, ON N6A 5C1, Canada \\ 3 Department of Biology, University of Western Ontario, London, ON N6A 5B7, Canada; \\ msoltys4@uwo.ca (M.P.M.S); dlee335@uwo.ca (D.K.L.); lachance@uwo.ca (M.-A.L.) \\ * Correspondence: bkaras@uwo.ca
}

Received: 10 November 2018; Accepted: 18 January 2019; Published: 23 January 2019

\begin{abstract}
Yeasts belonging to the Metschnikowia genus are particularly interesting for the unusual formation of only two needle-shaped ascospores during their mating cycle. Presently, the meiotic process that can lead to only two spores from a diploid zygote is poorly understood. The expression of fluorescent nuclear proteins should allow the meiotic process to be visualized in vivo; however, no large-spored species of Metschnikowia has ever been transformed. Accordingly, we aimed to develop a transformation method for Metschnikowia borealis, a particularly large-spored species of Metschnikowia, with the goal of enabling the genetic manipulations required to study biological processes in detail. Genetic analyses confirmed that M. borealis, and many other Metschnikowia species, are CUG-Ser yeasts. Codon-optimized selectable markers lacking CUG codons were used to successfully transform $M$. borealis by electroporation and lithium acetate, and transformants appeared to be the result of random integration. Mating experiments confirmed that transformed-strains were capable of generating large asci and undergoing recombination. Finally, random integration was used to transform an additional 21 yeast strains, and all attempts successfully generated transformants. The results provide a simple method to transform many yeasts from an array of different clades and can be used to study or develop many species for various applications.
\end{abstract}

Keywords: genome engineering; synthetic biology; yeasts; Metschnikowia; genetic tools; DNA delivery; CUG-Ser

\section{Introduction}

For decades, yeasts have proven to be tremendously useful in the study of life's fundamental processes. As single-celled models for eukaryotic life, they have short generation times, can be easily manipulated, and contain thousands of conserved genes-many of which are vitally important across nearly all living organisms [1,2]. While the discovery of new species provides us with new opportunities, genetic tools previously developed for Saccharomyces cerevisiae and other model organisms must be re-optimized for use in newly discovered species [3,4]. Altered membrane composition, modified gene expression, and alternative codon usage can prevent the seamless application of established transformation methods onto new species. Such transformation methods are crucial in the development of targeted knockouts, changes in gene expression, and fusion proteins that allow the study of biological processes in detail. 
Of particular interest among yeasts are the 81 or more species assigned to the genus Metschnikowia, some of which have become popular for their varied applications [5]. For example, M. pulcherrima is of importance to winemakers for its generation of pleasant aromas in high-quality wines, and its anti-microbial properties further its potential as an industrial microorganism [6,7]. Similarly, M. bicuspidata has been studied extensively for its role as a pathogen, and other species (including M. agaves and M. hawaiiensis) are being studied as potential anti-aging agents in cosmetics [5].

Metschnikowia species are particularly intriguing, due to the unusual formation of only two needle-shaped ascospores during their sexual cycle. A two-spored meiotic product stands in sharp contrast to the four spores arising from meiosis in many other yeast species (like S. cerevisiae) [5]. Although the occurrence of meiotic recombination has been demonstrated in some Metschnikowia species [8] the fate of the nuclei during meiosis remains unclear, and is yet to be elucidated. The expression of fluorescent nuclear fusion proteins should allow the meiotic process to be visualized in vivo, but the generation of such fusions is greatly hindered by the lack of genetic tools available for Metschnikowia species. M. pulcherrima is the only species ever to have been transformed [9]. Unfortunately, the small size of its ascospores and the difficulty of generating abundant asci make M. pulcherrima less than ideal for the study of meiotic nuclei.

In contrast, $M$. borealis is one of many Metschnikowia species that form ascospores that can reach 20-50 times the size of normal budding cells [10]. The giant, elongated spores would greatly facilitate the task of elucidating the meiotic process in Metschnikowia species. The initial selection of $M$. borealis for study is further justified by the fact that the species has the highest maximum growth temperature among the large-spored Metschnikowia species $\left(37^{\circ} \mathrm{C}\right)$, making it more likely to survive the high-temperature incubations required by many of the conventional yeast transformation methods [11,12]. Therefore, we aimed to develop and optimize a transformation method for M. borealis to enable the study of the species in more detail.

Our approach builds on the conventional methods used to transform other yeast species, such as S. cerevisiae and M. pulcherrima. After identifying antibiotics that can be used as selectable markers, we developed various genetic constructs carrying the appropriate genes to allow growth on selective plates. We then tested conventional transformation techniques, such as lithium acetate transformation and electroporation to introduce the various genetic cassettes [11]. It was originally anticipated that differences in membrane composition, antibiotic sensitivity, and origins of replication may pose significant obstacles in our ability to seamlessly apply classical transformation methods from S. cerevisiae onto M. borealis. Accordingly, we developed a systematic approach entailing a variety of different transformation methods, using a variety of different antibiotic resistance markers to develop a successful transformation method for $M$. borealis and other CUG-Ser yeasts.

\section{Materials and Methods}

\subsection{Microbial Strains and Growth Conditions}

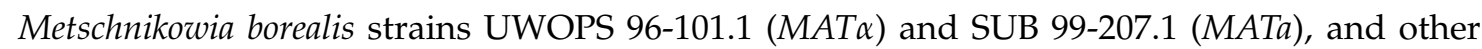
strains that were used in transformation experiments were obtained from the yeast collection of the Department of Biology, University of Western Ontario, where they are kept frozen in liquid nitrogen. Saccharomyces cerevisiae VL6-48 (ATCC MYA-3666: MAT $\alpha$ his3- $\Delta 200$ trp1- $\Delta 1$ ura3-52 lys2 ade2-1 met14 $\left.c i r^{0}\right)$. All strains were grown in YPAD broth ( $1 \%$ yeast extract, $2 \%$ peptone, $0.01 \%$ adenine hemisulfate, $2 \%$ D-glucose) at $30{ }^{\circ} \mathrm{C}$ with shaking at $225 \mathrm{rpm}$, with the exception of $C$. aff. bentonensis, M. bicuspidata and $\mathrm{M}$. orientalis, which were grown at $27^{\circ} \mathrm{C}$. Stationary cultures were grown on the same media containing $1 \%$ agar at the same growth temperatures.

\subsection{Codon-Optimization of KanMX and Sh ble}

To codon-optimize the KanMX and Sh ble selectable markers, each CTG codon was changed to a TTG, and the resulting gene sequence was uploaded to the OPTIMIZER tool 
(http:/ / genomes.urv.es/OPTIMIZER/) [13]. The codon frequencies from $M$. borealis in three highly expressed yeast genes- $A D H 1, T D H 3$, and ENO1-were used to guide optimization. A table was generated using the Codon Usage Database (https:/ / www.kazusa.or.jp/codon/) and entered into OPTIMIZER to guide codon-optimization. The method used for optimization was one amino acid to one codon, with a maximum of 25 allowed codon changes. Optimized genes were called MbKanMX and MbShBle.

\subsection{Yeast DNA Isolation}

Genomic DNA was isolated from $M$. borealis using a modified alkaline lysis method. Individual colonies were suspended in $1.0 \mathrm{~mL}$ of sterile double-deionized water $\left(\mathrm{sddH}_{2} \mathrm{O}\right)$ and pelleted at $3000 \times g$ for $5 \mathrm{~min}$. Cells were resuspended in $250 \mu \mathrm{L} \mathrm{P1} \mathrm{Buffer} \mathrm{(Qiagen,} \mathrm{Valencia,} \mathrm{CA,} \mathrm{USA),} 0.25 \mu \mathrm{L}$ of $14 \mathrm{M} \beta$-mercaptoethanol, and $12.5 \mu \mathrm{L}$ zymolyase solution $\left(10 \mathrm{mg} \mathrm{mL}^{-1}\right.$ zymolyase $20 \mathrm{~T}$ in $25 \%$ glycerol), and incubated at $37^{\circ} \mathrm{C}$ for $1 \mathrm{~h}$. Following incubation, $250 \mu \mathrm{L}$ of P2 Buffer (Qiagen) was added, gently mixed, and the suspension was incubated at room temperature for $5 \mathrm{~min}$. Subsequently, $250 \mu \mathrm{L}$ P3 Buffer (Qiagen) was added, gently mixed, and the suspension was incubated on ice for $10 \mathrm{~min}$. Cellular debris was pelleted at max speed for $10 \mathrm{~min}$ at $4{ }^{\circ} \mathrm{C}$, and the supernatant was transferred to a separate tube. Next, $750 \mu \mathrm{L}$ ice-cold isopropanol was added to the supernatant, and the mixture was frozen at $-80{ }^{\circ} \mathrm{C}$ for $15 \mathrm{~min}$. The DNA was pelleted at top speed for $10 \mathrm{~min}$ at $4{ }^{\circ} \mathrm{C}$, washed with $750 \mu \mathrm{L}$ ice-cold 70\% ethanol, and dissolved in $50 \mu \mathrm{L}$ Tris-EDTA (TE) pH 8.0.

\subsection{Lithium Acetate Transformations}

The lithium acetate transformations were conducted as described previously, with some modifications [14]. Briefly, a $50 \mathrm{~mL}$ cell culture was grown to an $\mathrm{OD}_{600}$ of 1.0 and pelleted at $6000 \times g$ for $5 \mathrm{~min}$. The pellet was washed 3 times with and resuspended in $1 \mathrm{~mL}$ sterile water. Next, $100 \mu \mathrm{L}$ of cells were transferred to a sterile tube and pelleted to remove the supernatant. The pellet was resuspended in $360 \mu \mathrm{L}$ of transformation mix (33\% PEG 3350 with $0.1 \mathrm{M} \mathrm{LiOAc}, 100 \mu \mathrm{g}$ salmon sperm carrier DNA, and $1 \mu \mathrm{g}$ DNA), and vortexed briefly. The mixture was incubated with shaking at $39^{\circ} \mathrm{C}$ and $300 \mathrm{rpm}$ for $30 \mathrm{~min}$, and then pelleted to remove the transformation mix. The pellet was resuspended in $1 \mathrm{~mL}$ of YPAD in a $1.5 \mathrm{~mL}$ Eppendorf tube, recovered for $2 \mathrm{~h}$ at $30^{\circ} \mathrm{C}$ and $225 \mathrm{rpm}$, plated on the appropriate selective media, and incubated at $30{ }^{\circ} \mathrm{C}$ for 2 days.

\subsection{Electroporation Conditions}

Electroporation experiments were conducted as previously described [15], with slight modifications. Briefly, cells were grown to an $\mathrm{OD}_{600}$ of 1.5 in $50 \mathrm{~mL}$ YPAD and pelleted at $5000 \times g$ for $5 \mathrm{~min}$. The pellet was resuspended in $10 \mathrm{~mL} 0.1 \mathrm{M} \mathrm{LiOAc}$ in $1 \times \mathrm{TE}, \mathrm{pH} 7.5$, and incubated with shaking at $150 \mathrm{rpm}$ and $30^{\circ} \mathrm{C}$ for $1 \mathrm{~h}$. Next, $250 \mu \mathrm{L} 1 \mathrm{M}$ DTT was added, and cells were returned to the incubator for $30 \mathrm{~min}$. After adding $40 \mathrm{~mL}$ ice-cold sterile water, cells were pelleted, washed with $25 \mathrm{~mL}$ ice-cold sterile water, washed again with $5 \mathrm{~mL}$ ice-cold $1 \mathrm{M}$ sorbitol, and resuspended in $250 \mu \mathrm{L} 1 \mathrm{M}$ sorbitol. Electro-competent cells were placed at $-80^{\circ} \mathrm{C}$ in $100 \mu \mathrm{L}$ aliquots. When needed, cells were quickly thawed in a $37^{\circ} \mathrm{C}$ water bath and immediately placed on ice. Electroporation reactions were prepared in $0.2 \mathrm{~cm}$ cuvettes with $40 \mu \mathrm{L}$ electro-competent cells, $5 \mu \mathrm{L}$ DNA $(1 \mu \mathrm{g})$, and $20 \mu \mathrm{g}$ single-stranded carrier DNA, and conditions were $1.8 \mathrm{kV}, 200 \Omega$, and $25 \mu \mathrm{F}$. Cells were collected from each cuvette in $1 \mathrm{~mL}$ of YPAD and allowed to recover in Eppendorf tubes for $2 \mathrm{~h}$ at $30^{\circ} \mathrm{C}$ and $225 \mathrm{rpm}$. For each transformation, $20 \mu \mathrm{L}$ were plated on the appropriate selective media and incubated at $30{ }^{\circ} \mathrm{C}$ for 2 days.

\subsection{Locating the Insertion Site}

Metschnikowia borealis cells were transformed with PCR-linearized CaNAT1 flanked by 60 base-pair sequences of homology to the HIS3 promoter and terminator, and DNA was isolated from 5 colonies by Alkaline Lysis as described above. DNA samples were digested with CfoI 
(Promega, Madison, WI, USA), with expected cut sites every 600 base-pairs in the M. borealis genome and no cut sites within CaNAT1. Digested samples were then ligated using T4 DNA Ligase (New England BioLabsWhitby, ON, Canada). Qiagen Multiplex (Cat No. 206143) was used to PCR-amplify the unknown sequences surrounding the insertion site, using primers that bind within CaNAT1 and that were oriented to amplify the surrounding sequence. PCR conditions were 95 ${ }^{\circ} \mathrm{C}$ melting for $30 \mathrm{~s}, 60{ }^{\circ} \mathrm{C}$ annealing for $90 \mathrm{~s}$, and $72{ }^{\circ} \mathrm{C}$ extension for $90 \mathrm{~s}$, cycled 35 times. PCR products were sent for sequencing at the DNA Sequencing Facility and Robarts Research Institute (London, ON, Canada). To identify insertion sites, sequences adjacent to the inserted marker were analyzed using BLASTn with Geneious 11.1.5 to search the M. borealis genome [16]. For secondary verification, primers were designed to anneal $\sim 150$ base-pairs upstream and downstream of the insertion site, and PCR was used to observe the insertion of the 693-base-pair cassette.

\subsection{Mating Experiments}

M. borealis MATa was transformed with MbShBle, and used in mating experiments with a MAT $\alpha$ strain that had been transformed with CaNAT1. MbShBle-transformed MATa was thinly spread on $2 \%$ agar plates containing $1 / 2$ strength glucose medium lacking histidine and uracil (Teknova, catalogue number C7221). CaNAT1-transformed MAT $\alpha$ was then spread perpendicular to $M A T a$, creating a grid with many sections where both strains were mixed. The cells were incubated for 2 days at room temperature. Approximately $20 \%$ of the cells were subsequently collected and resuspended in $25 \mathrm{~mL}$ of YPAD, and then grown at $30^{\circ} \mathrm{C}$ for $6 \mathrm{~h}$ with shaking at $225 \mathrm{rpm}$. Next, $1 \mathrm{~mL}$ aliquots of culture were plated on YPAD containing $200 \mathrm{mg} \mathrm{L}^{-1}$ zeocin and $75 \mathrm{mg} \mathrm{L}^{-1}$ nourseothricin and incubated at $30^{\circ} \mathrm{C}$. After two days, resulting colonies were collected, serially diluted, and plated on YPAD with $200 \mathrm{mg} \mathrm{L}^{-1}$ zeocin and $75 \mathrm{mg} \mathrm{L}^{-1}$ nourseothricin. Purified recombinant colonies were grown overnight in YPAD, serially diluted, and plated alongside wildtype $M A T a$, wildtype $M A T \alpha$, MbShBle-transformed MATa, and CaNAT1-transformed MATa.

\section{Results}

\subsection{Identification of Antibiotics that Inhibit Growth of M. borealis}

Before designing genetic cassettes (containing selection marker flanked by homology/promoter/terminator sequences) for transformation, it was necessary to identify antibiotics that inhibit the growth of $M$. borealis to enable us to choose appropriate antibiotic resistance markers. Cultures of $M$. borealis $M A T a$ and $M A T \alpha$ were grown to $\mathrm{OD}_{600}$ of 1.5 , pelleted, and resuspended to an $\mathrm{OD}_{600}$ of 3.0. Cultures were spot-diluted in $5 \mu \mathrm{L}$ aliquots on YPAD agar plates with various concentrations of geneticin, zeocin, or nourseothricin (Supplementary Table S1); and it was determined that each antibiotic was effective at inhibiting growth at 400,125 , and $50 \mathrm{mg} \mathrm{L}^{-1}$, respectively. To further prevent the occurrence of spontaneous colonies, higher concentrations of nourseothricin $\left(75 \mathrm{mg} \mathrm{L}^{-1}\right.$ or $\left.100 \mathrm{mg} \mathrm{L}^{-1}\right)$ and zeocin $\left(200 \mathrm{mg} \mathrm{L}^{-1}\right)$ were used for selection.

\subsection{Growth Rate of M. borealis}

Many conventional yeast transformation methods require that a culture to be grown to a specific optical density at $600 \mathrm{~nm}$; accordingly, it was necessary to determine the growth rate of M. borealis, to enable us to accurately calculate the growth time to the required optical density. Three cultures of each mating type of M. borealis (MATa and MAT $\alpha$ ) were grown to mid-log phase $\left(\mathrm{OD}_{600}=1.0\right)$, and diluted in $50 \mathrm{~mL}$ of YPAD to an optical density of 0.065 . Each culture was grown at $30{ }^{\circ} \mathrm{C}$ with shaking at $225 \mathrm{rpm}$, and $\mathrm{OD}_{600}$ was recorded at $1 \mathrm{~h}$ time points for $8 \mathrm{~h}$. The average $\mathrm{OD}_{600}$ of the three cultures for each mating type was recorded, and the doubling time was calculated as $76 \mathrm{~min}$ for mating type a and 74 min for mating type $\alpha$ (Supplementary Figure S1). 


\subsection{M. borealis Codon Usage}

A series of transformation attempts were made with $M$. borealis using genetic cassettes containing the NAT, KanMX, and Sh ble genes; however, no colonies were ever obtained for any of the transformation attempts. While investigating factors that would prevent us from obtaining colonies of $M$. borealis, the question of genetic code became significant. Some species (including M. bicuspidata and M. fructicola) have been reported to recognize a CUG codon as a serine (yeast alternative nuclear code) $[17,18]$, which fits within the current view that the Metschnikowiaceae belong to the CUG-Ser 1 clade of budding yeasts [19]. However, the only published literature regarding the genetic code of M. pulcherrima indicated that the species translates the CUG codon to leucine (standard code) [20]. The NAT, KanMX, and Sh ble cassettes used in the transformation attempts contain 8, 4, and 5 CUG codons in their open reading frames, respectively.

In an attempt to determine the genetic code of $M$. borealis, the coding sequence of URA3 from M. borealis was translated with the ExPASy translate tool, using both the standard code and yeast alternative nuclear code, and aligned with the 30 top hits from a URA3-BLASTx search using MUSCLE (Figure 1A). A leucine aligned at a highly conserved serine or threonine position when URA3 was translated with the standard genetic code; however, a serine aligned at that position when translated with the yeast alternative nuclear code.

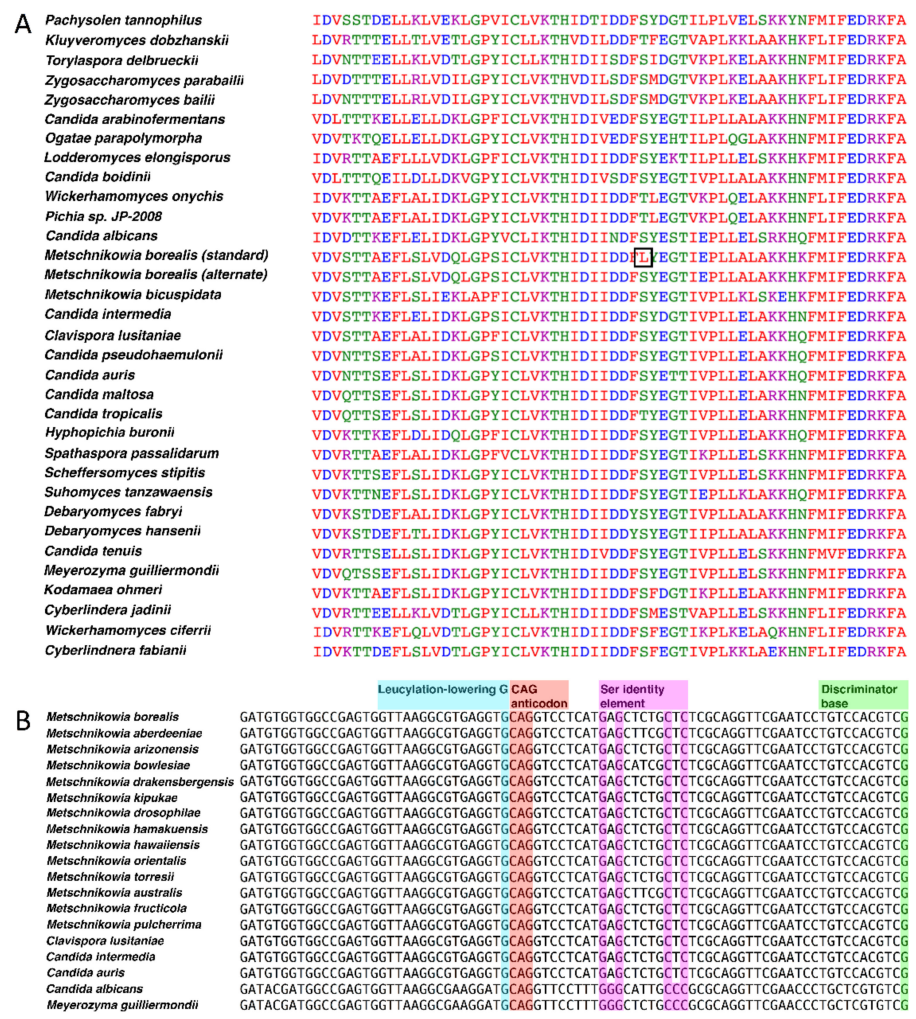

Figure 1. Bioinformatic analyses of the genetic code of $M$. borealis. (A) Sequence alignment of $U R A 3$ (encodes Orotidine 5'-phosphate decarboxylase). The coding sequence of URA3 from M. borealis was submitted as a query sequence to BLASTx, using the standard code for the predicted protein sequence. The protein sequences of the top 30 hits were aligned in MUSCLE with the M. borealis URA3 gene, translated in both the standard code and yeast alternative code using the ExPASy translate tool. A CUG codon aligned at a highly conserved serine or threonine amino acid position, producing a leucine when URA3 was translated using the standard code (black square). (B) tRNA $_{\mathrm{CAG}}{ }^{\text {Ser }}$ sequences aligned for many species of Metschnikowia and various CUG-Ser yeasts. Sequences were aligned in MEGA6 and annotated as described previously [18]. 
An examination of available Metschnikowiaceae genome sequences by tRNAscan-SE v2.0 [21] revealed that all species encoded at least one tRNA with a CAG anticodon. The retrieved tRNA sequences were analyzed through the tRNAscan-SE on-line web server, aimed at estimating the most probable translated amino acid. All Metschnikowia species that were examined had serine as the most probable translation with scores of 85 or more. Genome sequences for Candida aff. bentonensis or relatives are not available. Further evidence arose from aligning the sequence for RNAA $_{C A G}$ from $M$. borealis with the $\mathrm{tRNA}_{\mathrm{CAG}}{ }^{\mathrm{Ser}}$ of related CUG-Ser clade yeast species (Figure 1B). The tRNA $\mathrm{CAG}_{\text {from }}$ $M$. borealis and other Metschnikowiaceae contain the serine discriminator base and identity elements, indicating that the tRNAs identified are in fact tRNA $\mathrm{CAG}^{\text {Ser }}$. No tRNA $\mathrm{CAG}^{\text {Leu }}$ could be identified in any of the Metschnikowia species that were analyzed, including M. pulcherrima.

\subsection{Transformation of M. borealis with Codon-Optimized Cassettes}

Optimizer was used to codon-optimize the NAT, KanMX, and Sh ble genes for expression in $M$. borealis based on the codon frequencies in three highly expressed yeast genes: ADH1, TDH3, and ENO1. The optimized genes were translated with the ExPASy translate tool, using both the standard code and yeast alternative nuclear code, and aligned with BLAST to ensure that the same protein product would be produced. No differences were found between the wildtype gene products and the optimized gene products, regardless of whether the optimized genes were translated with the standard code or yeast alternative nuclear code. The optimized NAT gene was substantially similar to CaNAT1, a gene optimized for expression in Candida albicans [22]. Due to its previous success in transforming C. albicans, another CUG-Ser species, CaNAT1 was used in this experiment. Codon-optimized KanMX and Sh ble genes were called MbKanMX and MbShble.

Optimizer was used to codon-optimize the NAT, KanMX, and Sh ble genes for expression in $M$. borealis based on the codon frequencies in three highly expressed yeast genes: ADH1, TDH3, and ENO1. The optimized genes were translated with the ExPASy translate tool, using both the standard code and yeast alternative nuclear code, and aligned with BLAST to ensure that the same protein product would be produced. No differences were found between the wildtype gene products and the optimized gene products, regardless of whether the optimized genes were translated with the standard code or yeast alternative nuclear code. The optimized NAT gene was substantially similar to CaNAT1, a gene optimized for expression in Candida albicans [22]. Due to its previous success in transforming C. albicans, another CUG-Ser species, CaNAT1 was used in this experiment. Codon-optimized KanMX and Sh ble genes were called MbKanMX and MbShble.

The second set of antibiotic resistance genes were created by reintroducing the same 8,4 , and 5 CUG codons found in the wildtype NAT, KanMX, and Sh ble genes, respectively, as a control for codon usage. All six codon-optimized genes (three in standard code and three in yeast alternative nuclear code) were ordered as separate G-block fragments from Integrated DNA Technologies. All genes were amplified with primers containing homologous hooks to the M. borealis ADH1 promoter and terminator and were used to transform the species by electroporation. Transformants were plated on YPAD with appropriate concentrations of each antibiotic $\left(75 \mathrm{mg} \mathrm{L}^{-1}\right.$ nourseothricin for CaNAT1, $200 \mathrm{mg} \mathrm{L}^{-1}$ zeocin for MbShBle, and $400 \mathrm{mg} \mathrm{L}^{-1}$ geneticin for MbKanMX) alongside negative controls. Several colonies appeared for all samples that were transformed using the yeast alternative nuclear code, but no colonies appeared on any of the other plates (Figure 2). Similar results were seen using our best lithium acetate protocol. Selected transformants from the electroporation experiment were verified by genotyping (Supplementary Figure S2). Transformation efficiencies were determined for each method by transforming MATa and MAT $\alpha$ by each method in triplicate, and were calculated to be approximately 1600 and 817 CFUs per $\mu \mathrm{g}$ of DNA for electroporation, and 9 and 4 CFUs per $\mu \mathrm{g}$ of DNA for lithium acetate transformation, respectively (Supplementary Table S2).

The relatively high efficiency of electroporation was believed to be attributable to homologous recombination, because each cassette contained by 60 base-pairs of homology to the $A D H 1$ promoter and terminator. Based on this assumption, we attempted to generate histidine auxotrophs by transforming 
M. borealis with CaNAT1 flanked by 60 base-pair sequences of the HIS3 promoter and terminator. After transformation, ten colonies of each making type were spot-diluted and re-isolated, but all grew on media lacking histidine. Subsequent genotyping by PCR identified the presence of both HIS3 and CaNAT1, but failed to obtain bands across the HIS3 promoter and terminator junctions with CaNAT1: Bands that would be expected in the case of a successfully targeted knockout (Supplementary Figure S3). Despite the random integration of our cassettes, double marker transformation experiments revealed that two cassettes were inserted in approximately $2 \%$ of transformants (Supplementary Table S3).

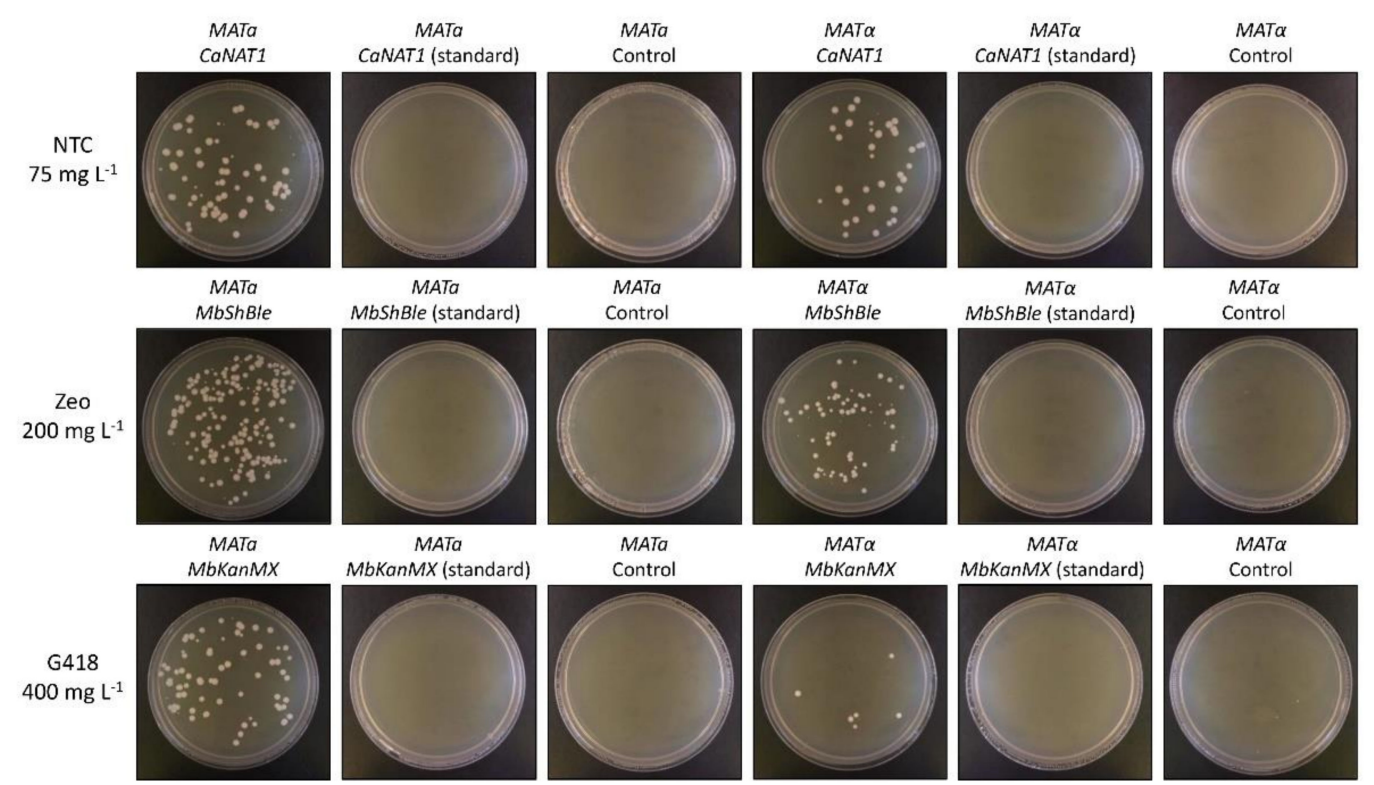

Figure 2. Electroporation of M. borealis with CaNAT1, MbKanMX, and MbShBle in standard and yeast alternative nuclear code. Electro-competent cells of $M$. borealis (MATa and MAT $\alpha$ ) were transformed with $1 \mu \mathrm{g}$ of PCR-amplified CaNAT1, MbShBle, or MbKanMx, in standard or alternative code, flanked by 60 base-pair sequences of the $M$. borealis $A D H 1$ promoter and terminator, using the electroporation protocol outlined in this study. After recovery, $20 \mu \mathrm{g}$ of each transformation was plated on YPAD with the appropriate antibiotic and incubated at $30^{\circ} \mathrm{C}$ for two days. NTC = nourseothricin; Zeo = zeocin; G418 = geneticin.

\subsection{Location of Insertion Site}

Subsequent screening of more than 30 MATa and 30 MAT $\alpha$ transformants for HIS3 knockouts failed to identify any successful insertions in the HIS3 gene. To identify the correct insertion sites, DNA was isolated from 6 MAT $\alpha$ transformants, digested with CfoI, circularized with T4 ligase, and the surrounding genomic DNA was PCR-amplified with primers binding within the inserted CaNAT1 cassette (oriented to amplify the surrounding sequence) (Supplementary Figure S3). We were successful in obtaining a PCR-fragment from the third MAT $\alpha$ transformant (clone 3), and the amplified gDNA was sequenced. Analysis with BLASTn indicated that the CaNAT1 cassette was inserted in scaffold bor-_s886 of M. borealis UWOPS 96-101.1, as annotated in GenBank (PRJNA312754), just upstream of the gene for peroxin-14. PCR was used to amplify this site in wildtype $M$. borealis, as well as all six isolated transformants, and the insertion was only observed at this site in clone 3.

\subsection{Mating Experiments}

To confirm that transformed-strains of $M$. borealis MATa and MAT $\alpha$ were capable of successfully mating, MbShBle-transformed MATa and CaNAT1-transformed MAT $\alpha$ were mated to develop ascospores (Figure 3A). Recombinant cells that contain both markers were isolated by serial dilution and genotyped to confirm that they contain both MbShBle and CaNAT1 (Figure 3B). 
Mated cells were then plated alongside wildtype MATa and MAT $\alpha, M b S h B l e$-transformed MATa, and CaNAT1-transformed MAT $\alpha$ on YPAD, YPAD with zeocin, YPAD with nourseothricin, and YPAD with nourseothricin and zeocin for comparison (Figure 3C).

A

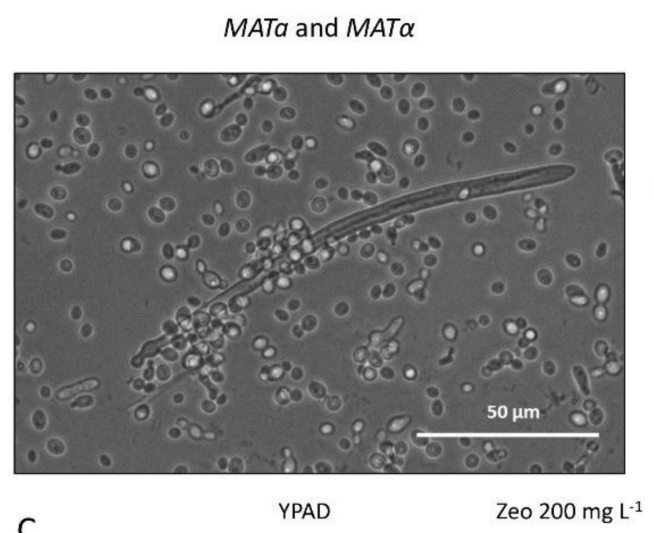

C

\begin{abstract}
YPAD
\end{abstract}

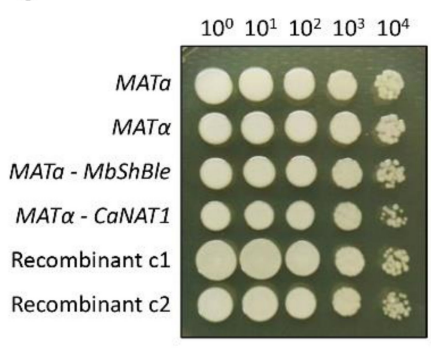

B

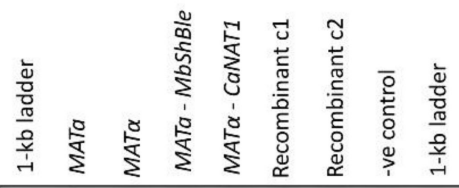

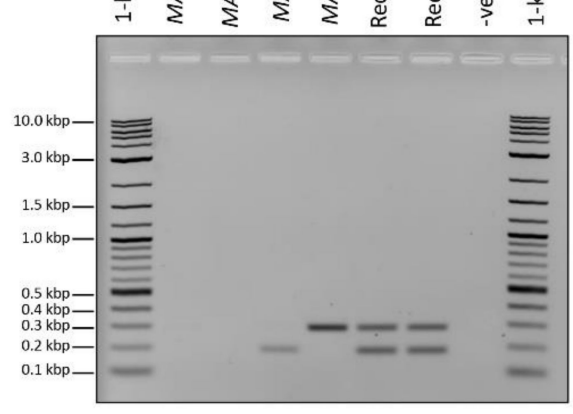

Zeo $200 \mathrm{mg} \mathrm{L}^{-1}$

NTC $75 \mathrm{mg} \mathrm{L}^{-1}$

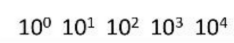

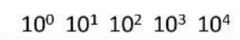

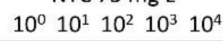
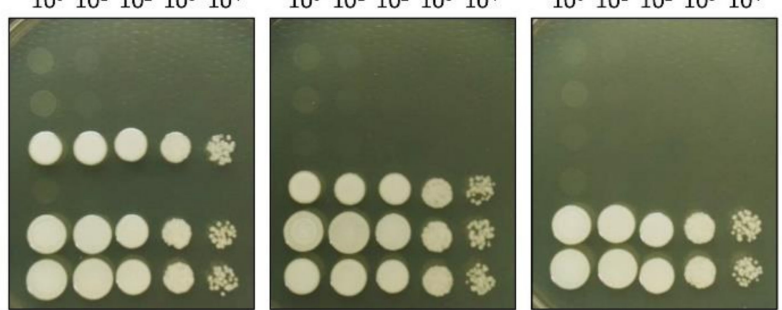

Figure 3. Mating experiments with marker-transformed M. borealis. (A) Photograph of a large mating ascospore. MbShBle-transformed MATa and CaNAT1-transformed MAT $\alpha$ were mated for two days on 1/2-strength glucose broth lacking histidine and uracil (Teknova), collected in YPAD, and photographed under a light microscope. (B) Genotyping results of marker-transformed diploids. Recombinant colonies harbouring both selectable markers were purified by spot dilution on YPAD with $200 \mathrm{mg} \mathrm{L}^{-1}$ zeocin and $75 \mathrm{mg} \mathrm{L}^{-1}$ nourseothricin. Recombinant colonies were genotyped by Qiagen multiplex with primers that amplify the CaNAT1 and MbShBle genes, alongside marker-transformed haploid strains and untransformed controls. MbShBle product size (C) Spot-dilutions of mated-colonies alongside transformed haploid strains and untransformed controls on YPAD, YPAD with $200 \mathrm{mg} \mathrm{L}^{-1}$ zeocin, YPAD with $75 \mathrm{mg} \mathrm{L}^{-1}$ nourseothricin, and YPAD with $200 \mathrm{mg} \mathrm{L}^{-1}$ zeocin and $75 \mathrm{mg} \mathrm{L}^{-1}$ nourseothricin. Plates were incubated at $30^{\circ} \mathrm{C}$ for two days. NTC = nourseothricin; Zeo = zeocin; G418 = geneticin.

\subsection{Transformation of other CUG-Ser Yeasts}

To determine if our electroporation method would yield transformants of other yeasts by random integration, we transformed strains of 21 yeast species with CaNAT1 cassettes (in standard and yeast alternative nuclear code) flanked by the same 60-base-pair minimal ADH1 promoter and terminator from $M$. borealis (Figure 4; Supplementary Table S4). Transformants were plated on YPAD with 75-200 $\mathrm{mg} \mathrm{L}^{-1}$ nourseothricin and incubated at $27-30{ }^{\circ} \mathrm{C}$ for $24-72 \mathrm{~h}$ until colonies appeared. All strains yielded colonies for the alternative-coded CaNAT1, but only S. cerevisiae and Candida aff. bentonensis yielded colonies when transformed with standard-coded CaNAT1 (Figure 4; Supplementary Table S4). It's important to note that the alternative-coded CaNAT1 lacks CUG codons and is therefore degenerate between the standard code and yeast alternative code; however, the standard-coded CaNAT1 uses CUG codons to translate leucine and is only translatable to CUG-Leu yeasts. 


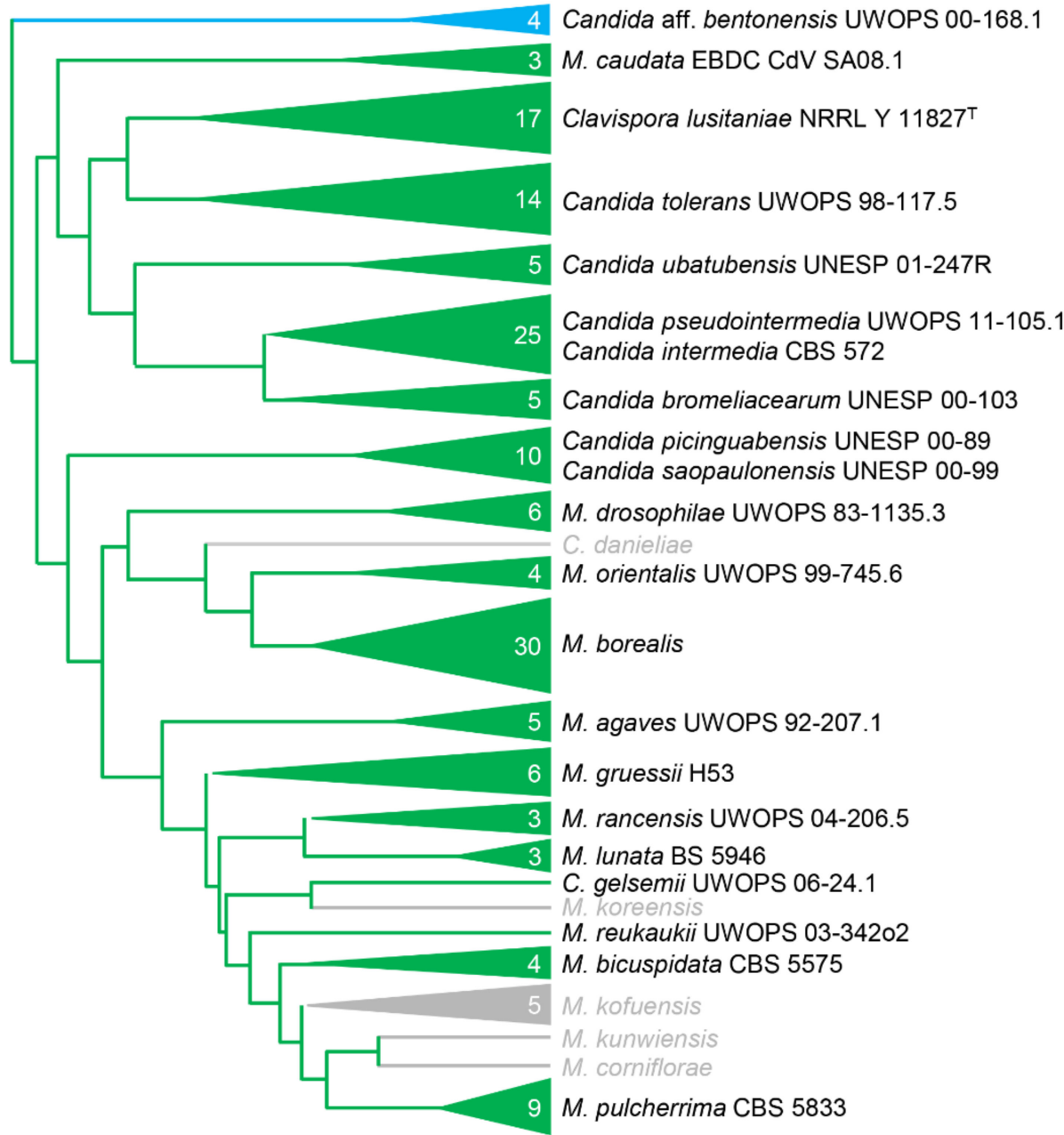

Figure 4. A phylogeny of the Metschnikowiaceae, modified from Lachance (2016) [5], showing the taxonomic range of successful transformations in the family. Representative strains were successfully transformed with only the alternative-coded CaNAT1 construct (green) or with both the alternative-coded and the standard-coded CaNAT1 (blue) construct. Species in grey were not examined.

\section{Discussion}

Through pioneering experiments, we were successful in determining the necessary conditions to transform Metschnikowia borealis, and the method was effective in many other yeast species. We were able to identify the $\mathrm{RNAA}_{\mathrm{CAG}}{ }^{\mathrm{Ser}}$ in M. borealis, as well as many other Metschnikowia species, and transformation experiments confirmed that many other-if not all-Metschnikowia species are also CUG-Ser 1 clade yeasts (Figures 2B and 4). It is unclear why M. pulcherrima was previously reported to be a CUG-Leu yeast [9].

Once we determined that $M$. borealis utilizes the yeast alternative nuclear code, the species was successfully transformed with selectable markers that contain homologous hooks to the HIS3 promoter and terminator, with the high efficiency. Transformants were first believed to have been obtained through a targeted knockout of HIS3 by homologous recombination; however, all colonies grew on medium lacking histidine, and genotyping confirmed the presence of both the selectable marker and the wildtype HIS3 gene. Additionally, we failed to observe any of the expected genotyping bands associated with the knockout junctions. This indicated that the cassettes were not incorporated into the genome by targeted integration, but by another mechanism with high efficiency.

Although we only identified and confirm one insertion site (in clone 3), the HIS3-targeted cassette was found to insert in the site just upstream of the peroxin-14 gene in clone 3, and not in any of the 
other transformants tested (Supplementary Figure S2C). Therefore, the cassette must have inserted in different locations in the other transformants, indicating that M. borealis appears to integrate DNA cassettes randomly into its genome. Even though the species is not efficient at targeted integration, its high efficiency at random integration makes it simple to transform and incorporate exogenous sequences into the genome.

Finally, our electroporation transformation method was successful across all CUG-Ser yeasts that were attempted, as well as S. cerevisiae and Candida aff. bentonensis. This confirms the suspicion [5] that $C$. bentonensis is not a member of the Metschnikowiaceae. The results of this study indicate that the species is in fact a CUG-Leu yeast. Fortunately, the CaNAT1, MbShBle, and MbKanMX genes used in our experiments lack the use of a CUG codon and are degenerate to the standard and yeast alternative nuclear code. Therefore, the markers would likely be universal to all CUG-Leu, CUG-Ser, and CUG-Ala yeasts [19].

Through the use of random integration, the methods outlined in this study make it possible to transform a large range of yeasts and would likely be successful in numerous additional species in a wide array of clades. Random integration can be used to introduce elements to many understudied yeast species to enable their study in more detail. In particular, it is now possible to design codon-optimized fluorescent nuclear-fusion proteins that can be used to elucidate the unusual meiotic process in Metschnikowia species that leads to the production of only two ascospores from a diploid zygote.

Supplementary Materials: The following are available online at http:/ www.mdpi.com/2073-4425/10/2/78/s1, Figure S1: Growth rate of M. borealis MATa and MAT $\alpha$, Figure S2: Genotyping transformants, Figure S3: Identification of insertion sites; Table S1: Identification of antibiotic sensitivity, Table S2: Transformation efficiencies for M. borealis, Table S3: Double marker transformation of M. borealis, Table S4: Transformation results for additional yeast strains.

Author Contributions: Conceptualization: Z.B.G., M.P.M.S., P.J., M.-A.L., B.J.K.; methodology, Z.B.G., M.P.M.S., C.L., J.A.T., R.S.M., C.L., M.A., D.K.L., P.J., M.-A.L., B.J.K; validation, Z.B.G., M.P.M.S., C.L., J.A.T., R.S.M., C.L., M.A., D.K.L., P.J., M.-A.L., B.J.K; formal analysis, Z.B.G., M.P.M.S., C.L., J.A.T., R.S.M., C.L., M.A., D.K.L., P.J., M.-A.L., B.J.K; investigation, Z.B.G., M.P.M.S., C.L., J.A.T., R.S.M., C.L., M.A., D.K.L., P.J., M.-A.L., B.J.K; resources, M.-A.L., B.J.K; data curation, Z.B.G., M.P.M.S., C.L., J.A.T., R.S.M., C.L., M.A., D.K.L., P.J., M.-A.L., B.J.K; writing-original draft preparation, Z.B.G., M.P.M.S., R.S.M., M.A., M.-A.L., B.J.K; writing-review and editing, Z.B.G., M.P.M.S., R.S.M., M.A., M.-A.L., B.J.K; visualization, Z.B.G., M.P.M.S., C.L., J.A.T., R.S.M., C.L., M.A., D.K.L., P.J., M.-A.L., B.J.K; supervision, Z.B.G., P.J., M.-A.L., B.J.K; project administration M-A.L., B.J.K; funding acquisition, M.-A.L., B.J.K.

Funding: This research was funded by: Designer Microbes Inc.; NSERC Experience grants to Z.B.G., C.L., C.L., R.S.M. The B.J.K. lab is also supported by Natural Sciences and Engineering Research Council of Canada (NSERC), grant number: RGPIN-2018-06172.

Conflicts of Interest: B.J.K. is Chief Executive Officer of Designer Microbes Inc. B.J.K., P.J. and M-A.L. hold Designer Microbes Inc. stock.

\section{References}

1. Deng, J.; Deng, L.; Su, S.; Zhang, M.; Lin, X.; Wei, L.; Minai, A.A.; Hassett, D.J.; Lu, L.J. Investigating the predictability of essential genes across distantly related organisms using an integrative approach. Nucl. Acids Res. 2011, 39, 795-807. [CrossRef] [PubMed]

2. Zhang, R.; Ou, H.-Y.; Zhang, C.-T. DEG: A database of essential genes. Nucl. Acids Res. 2004, 32, D271-D272. [CrossRef] [PubMed]

3. De Backer, M.D.; Maes, D.; Vandoninck, S.; Logghe, M.; Contreras, R.; Luyten, W.H. Transformation of Candida albicans by electroporation. Yeast Chichester Engl. 1999, 15, 1609-1618. [CrossRef]

4. Yildirim, S.; Thompson, M.G.; Jacobs, A.C.; Zurawski, D. V.; Kirkup, B.C. Evaluation of parameters for high efficiency transformation of Acinetobacter baumannii. Sci. Rep. 2016, 6, 22110. [CrossRef] [PubMed]

5. Lachance, M.-A. Metschnikowia: Half tetrads, a regicide and the fountain of youth. Yeast Chichester Engl. 2016. [CrossRef] [PubMed] 
6. González-Pombo, P.; Pérez, G.; Carrau, F.; Guisán, J.M.; Batista-Viera, F.; Brena, B.M. One-step purification and characterization of an intracellular beta-glucosidase from Metschnikowia pulcherrima. Biotechnol. Lett. 2008, 30, 1469-1475. [CrossRef] [PubMed]

7. Macwilliam, I.C. A survey of the antibiotic powers of yeasts. J. Gen. Microbiol. 1959, 21, 410-414. [CrossRef]

8. Marinoni, G.; Piškur, J.; Research, M.L.-F. Ascospores of large-spored Metschnikowia species are genuine meiotic products of these yeasts. FEMS Yeast Res. 2003, 3, 85-90. [CrossRef] [PubMed]

9. Nigro, F.; Sialer, M.M.F.; Gallitelli, D. Transformation of Metschnikowia pulcherrima 320, biocontrol agent of storage rot, with the Green Fluorescent Protein gene. J. Plant Pathol. 1999, 81, 205-208.

10. Lachance, M.-A.; Hurtado, E.; Hsiang, T. A stable phylogeny of the large-spored Metschnikowia clade. Yeast Chichester Engl. 2016, 33, 261-275. [CrossRef] [PubMed]

11. Kawai, S.; Hashimoto, W.; Murata, K. Transformation of Saccharomyces cerevisiae and other fungi. Bioeng. Bugs 2010, 1, 395-403. [CrossRef] [PubMed]

12. Lachance, M.-A.; Rosa, C.A.; Starmer, W.T.; Schlag-Edler, B.; Baker, J.S.F.; Bowles, J.M. Metschnikowia continentalis var. borealis, Metschnikowia continentalis var. continentalis, and Metschnikowia hibisci, new heterothallic haploid yeasts from ephemeral flowers and associated insects. Can. J. Microbiol. 1998, 44, 279-288. [CrossRef]

13. Puigbò, P.; Guzmán, E.; Romeu, A.; Garcia-Vallvé, S. OPTIMIZER: A web server for optimizing the codon usage of DNA sequences. Nucl. Acids Res. 2007, 35, W126-W131. [CrossRef] [PubMed]

14. Gietz, R.D. Yeast transformation by the LiAc/SS carrier DNA/PEG method. Methods Mol. Biol. Clifton 2014, 1205, 1-12. [CrossRef]

15. Froyd, C.A.; Kapoor, S.; Dietrich, F.; Rusche, L.N. The deacetylase Sir2 from the yeast Clavispora lusitaniae lacks the evolutionarily conserved capacity to generate subtelomeric heterochromatin. PLoS Genet. 2013, 9, e1003935. [CrossRef] [PubMed]

16. Kearse, M.; Moir, R.; Wilson, A.; Stones-Havas, S.; Cheung, M.; Sturrock, S.; Buxton, S.; Cooper, A.; Markowitz, S.; Duran, C.; et al. Geneious Basic: An integrated and extendable desktop software platform for the organization and analysis of sequence data. Bioinform. Oxf. Engl. 2012, 28, 1647-1649. [CrossRef] [PubMed]

17. Mühlhausen, S.; Kollmar, M. Molecular phylogeny of sequenced saccharomycetes reveals polyphyly of the alternative yeast codon usage. Genome Biol. Evol. 2014, 6, 3222-3237. [CrossRef] [PubMed]

18. Riley, R.; Haridas, S.; Wolfe, K.H.; Lopes, M.R.; Hittinger, C.T.; Göker, M.; Salamov, A.A.; Wisecaver, J.H.; Long, T.M.; Calvey, C.H.; et al. Comparative genomics of biotechnologically important yeasts. Proc. Natl. Acad. Sci. USA 2016, 113, 9882-9887. [CrossRef] [PubMed]

19. Krassowski, T.; Coughlan, A.Y.; Shen, X.-X.; Zhou, X.; Kominek, J.; Opulente, D.A.; Riley, R.; Grigoriev, I. V.; Maheshwari, N.; Shields, D.C.; et al. Evolutionary instability of CUG-Leu in the genetic code of budding yeasts. Nat. Commun. 2018, 9, 1887. [CrossRef] [PubMed]

20. Sugita, T.; Nakase, T. Non-universal usage of the leucine CUG codon and the molecular phylogeny of the genus Candida. Syst. Appl. Microbiol. 1999, 22, 79-86. [CrossRef]

21. Lowe, T.M.; Chan, P.P. tRNAscan-SE On-line: Integrating search and context for analysis of transfer RNA genes. Nucl. Acids Res. 2016, 44, W54-W57. [CrossRef] [PubMed]

22. Shen, J.; Guo, W.; Köhler, J.R. CaNAT1, a heterologous dominant selectable marker for transformation of Candida albicans and other pathogenic Candida species. Infect. Immunity 2005, 73, 1239-1242. [CrossRef] [PubMed]

(C) 2019 by the authors. Licensee MDPI, Basel, Switzerland. This article is an open access article distributed under the terms and conditions of the Creative Commons Attribution (CC BY) license (http:// creativecommons.org/licenses/by/4.0/). 